
\title{
28 Research Square \\ The Dignity of Burn Patients: A Qualitative Descriptive Study of Nurses and Caregivers
}

\section{Banafsheh Tehranineshat}

Shiraz University of Medical Sciences https://orcid.org/0000-0002-2066-5689

Mahnaz Rakhshan ( $D$ mzrakhshan@gmail.com )

https://orcid.org/0000-0003-1687-5154

\section{Camellia Torabizadeh}

Shiraz University of Medical Sciences

\section{Mohammad Fararouei}

Shiraz University of Medical Sciences

\section{Mark Gillespie}

university of Scotland

\section{Research article}

Keywords: Burns, Patients, Human dignity, Nursing, Family caregivers, Qualitative research

Posted Date: July 30th, 2020

DOl: https://doi.org/10.21203/rs.3.rs-17756/v3

License: (c) (1) This work is licensed under a Creative Commons Attribution 4.0 International License. Read Full License 


\section{Abstract}

Background: The essence of nursing care is preservation of patients' human dignity. The aggressive nature of severe burn injuries makes it hard to respect burn patient's existential dimensions and dignity. However, not much research has been conducted on respect for the dignity of burn patients.

Purpose: The present study aims to outline the concept of maintaining the dignity of burn patients from the perspective of their caregivers.

Methods: The study has a descriptive, qualitative research design. Data were collected using semistructured, in-depth, individual interviews. Data analysis was executed using conventional content analysis. The subjects were nurses and family caregivers from the biggest burns hospital in the southeast of Iran. The participants were selected via purposeful sampling $(n=20)$ until data saturation was reached. The study lasted from October 2017 to August 2018.

Results: Three main themes emerged from the information obtained from the interviews: creating a friendly atmosphere, showing respect, and providing comprehensive support.

Conclusion: Burn patients need to receive care in a friendly atmosphere where they are looked after by empathetic caregivers who give enough time to them and allow them to express their feelings and concerns. Also, patients' human values and beliefs should be respected and all aspects of their existence should be taken into account for their dignity to be preserved. Workshops designed based on the findings of the present study can help improve the quality of nursing care.

\section{Background}

Burn injury is one of the leading causes of morbidity and mortality worldwide [1]. Individuals from all age groups and various socioeconomic levels are liable to suffer from burns. The clinical experiences of burn survivors suggest that the condition is accompanied by severe distress and may lead to emotional, psychological, and physical changes in the victims [2]. Several studies have shown that burn survivors face various physical and psychological challenges during recovery which may affect all aspects of their lives [3].

The aggressive nature of severe burns and the heavy burden of having to deal with the consequences, including impaired physical functioning, disfigurement, and psychological distress, are a threat to the victims' human dignity and successful return to a normal life [4]. Experiencing unbearable pain, impaired performance, disfigurement, and changes in their self-image and social role affect burn patients' existence. All of the above-mentioned factors are positively associated with burn patients' social isolation, feelings of over-dependence on others, and fear of losing their dignity [2]. 
A basic duty of nurses in the burns wards of hospitals is to provide compassionate and respectful care to the patients [5]. According to Watson et al. (2011), caring is an ethical ideal in nursing which aims to maintain and improve human dignity-the goal of nursing is to help individuals achieve higher levels of mind-body-soul coordination. This goal can be realized through human-human caring processes and interactions, which, in turn, result in self-healing and self-controlling [6]. Confirming Watson's viewpoint, Roach states that humanity and human dignity are inseparable. On the same note, Jacobs points out that the essence of nursing is not to restore health or re-establish a holistic balance and coordination, but to show respect for human dignity [7].

Dignity is an important aspect of nursing care [7]. Preservation of human dignity is a core principle which applies not only to patients, but to all human beings [8]. In codes of ethics, respect for human dignity is the right of every human being and is regarded as an ethical duty of nurses. Human dignity is the perception of respect and competence in others: it allows a person to feel value and respect for others and believe that they can grow and develop [9].

Preservation of dignity is one of the most important ethical responsibilities of caregivers [10]. Protection of dignity is associated with increased patient satisfaction and self-esteem, reduced length of hospital stay, patients' enhanced ability to cope with illness, and giving patients the sense that their lives are meaningful [8]. Respect for human dignity enhances caregivers' motivation to provide quality care, resulting in an effective relationship between patients and caregivers [11]. In contrast, violation of dignity leads to psychological and spiritual distress, reduced motivation to survive, and deterioration of patients' physical and mental health [12]. In recent years, many studies have addressed patients' and caregivers' perceptions of human dignity [12]. In the literature, the nature of dignity has been assessed in several groups of patients and caregivers, including patients with cardiovascular diseases [13-14], terminally ill patients [15-16], elderly patients [17-18], hospitalized adolescents [19], autistic teenagers [10], and cancer patients[20-22]. However, our understanding of the ethical aspects of caring for burn patients is limited [23] and, despite the serious psychological, social, cultural and religious consequences of burns, the dignity of burn patients has not been explored yet [2]. Burn injuries and the ensuing complications may cause the affected to have a sense of inferiority, feel that their dignity is at risk, and impair their selfawareness [24].

Dignity is a concept defined based on cultural contexts and physical environments [25]. Clarifying the meaning of burn patient's dignity is crucial to fully respecting and preserving the dignity of this group. Therefore, it is necessary to establish the meaning of dignity and its related factors in this group of patients. Due to the abstract nature and complexity of the concept of dignity [8,26], a qualitative study is required to achieve a clear and deep knowledge of the concept [27]. A qualitative approach helps to discover problems related to burns and achieve an extensive understanding of burn patients' issues [2]. Research into burn patients' dignity can help with determining the various dimensions of this concept, identifying the effective factors in maintaining the dignity of burn patients, and providing quality care. Nurses' and family caregivers' descriptions of the dignity of burn patients can yield a better understanding of the concept in question in the context of caring for burn patients. The present study 
can help healthcare administrators and caregivers to provide a supportive and empowering environment where burn patients' rights and dignity are maintained. The present study aims to determine the concept of burn patients' dignity from the perspective of their caregivers.

Specific objectives

1. What are the experiences of nurses and family caregivers regarding burn patients' dignity?

2. Based on their experiences, how do nurses and family caregivers describe burn patients' dignity?

\section{Methods}

The present study is a descriptive qualitative work of research which uses a content analysis approach. Qualitative content analysis is a systematic qualitative research technique used to code and categorize data to extract a large amount of textual information to determine patterns in words, their relationships, structures, and discourses [28]. Qualitative research is a systematic mental process whose purpose is to describe human experiences and understandings [29]. As dignity is a concept or mental experience [8], the present study uses a qualitative exploratory design. The aim of the study is to explore the meaning of burn patients' dignity via conventional content analysis. According to Hsieh and Shannon, conventional content analysis applies when literature on the phenomenon under study is limited. When this is the case, researchers avoid using a preconceived understanding of the topic and, instead, try to have a definition of the topic emerge from data [29].

\section{Participants and research context}

A total of 14 nurses and 6 family caregivers were selected from the largest burn hospital in the south-east of Iran using purposeful sampling. The study lasted from October 2017 to August 2018. In the present study, the first researcher (BT) interviewed caregivers who had rich experience in the field of caring for burn patients, could provide the most information with respect to the research question, and were able to eloquently express their experiences. The inclusion criteria for the nurses were: having at least a bachelor's degree, a minimum of 6 months' work experience, experience of caring for a second-degree or third-degree burn patient, not being fatigued due to work overload in the hospital, being willing to participate in the study, being able to provide meaningful and rich information about the subject of the study, and speaking and understanding Persian. The inclusion criteria for the family caregivers were: being aged 18 years or above, being a member of the patient's family, e.g. a spouse, child, sibling, or a friend of the patient, not having a psychological or metabolic disorder to their knowledge, not being on medication which affects the mind, not suffering from physical or psychological fatigue due to caring for their patients, and being mentally and physically fit to be interviewed and experience of caring for a second-degree or third-degree burn patient. Also, to be included, family caregivers should be willing to participate in the study, be able to provide meaningful and rich information about the subject of the study, and speak and understand Persian. 
On the research team, the first three authors had a Ph.D. in nursing and experience of educational-clinical practice in burns departments. The second and third authors had previously conducted research in the area of patient dignity $[10,26]$. The present study was conducted to plug the gap in the existing literature on the dignity of burn patients. All the researchers were trained and skilled in qualitative research. The research context was Amiralmomenin Burn and Plastic Surgery Hospital, the largest burns facility in Shiraz, located in the south-east of Iran. This hospital offers all kinds of medical services related to burn injuries in the south of Iran. All the wards of this educational hospital provide specialized care in the fields of burns, rehabilitation, and plastic surgery.

The research setting comprised of all the sections of the above-mentioned hospital, including the male surgical ward, the female surgical ward, the emergency department, and ICU. Data were collected using face-to-face, semi-structured interviews with 14 nurses and 6 family caregivers.

\section{Data collection}

Due to lack of a specific protocol for recognizing the dignity of burn patients (in hospitals) in Iran, a comparative approach was adopted to identify suitable participants. The first author/interviewer selected the potential participants from different wards and work shifts based on her experience and informed judgment and the inclusion criteria. Only the subjects who had enough knowledge and experience in the area of burn patients' dignity were interviewed.

Informed consent was obtained from all the participants. The interviews were conducted by the first researcher (BT), with the permission of the ward's head nurse, in the nurses' break room. During the interviews, none of the other staff members were allowed to enter the room without permission.

In order to focus on the dimensions of burn patients' dignity, the first author (BT) initially interviewed a nurse and a family caregiver (not actual participants) using the final interview guideline. The interview guideline was oriented around the subjects' perceptions and definitions of burn patients' dignity, as well as their past and present experiences. The first author (BT), in order to encourage the participants to answer her questions openly and sincerely, informed the participants that she was a university faculty member, all information would remain confidential and anonymous, and participation was on a voluntary basis. Before each interview, the participants were introduced to the objectives and method of the study and the fact that the sponsorship was non-commercial.

The interviews started with a general question-"what does burn patients' dignity mean to you?"-to allow the participants to fully describe their understanding and experiences. Then, based on the participants' responses, more specific questions were asked to gather information more directly related to the objectives of the study, including "What experiences have you had which are related to the dignity of burn patients?" and "How do you feel after a patient's dignity is respected or ignored?". To get clearer answers, the interviewer used open-ended questions, such as "What do you mean by that?", "Can you explain further?", and "Can you give an example?". 
All the interviews were conducted and recorded by the first author (BT). The participants' voices were recorded using a Sony Voice Recorder ICD-TX650.

The duration of the interviews varied from 45 to 70 minutes. During the interviews, the participants' nonverbal communication was also noted. The participants were selected via purposeful sampling which continued until data saturation. Data saturation has been reached when no new categories can be extracted from the data and all the categories are saturated in terms of feature and dimension. In the present study, data saturation was reached after 16 interviews, and two further interviews were done to ensure that no new information could be extracted from the data.

\section{Data Analysis}

As data were being collected, they were analyzed using Graneheim and Lundman's approach to qualitative content analysis (2004). The approach consists of the following steps: transcribing the interviews immediately after completion, reading the transcripts to reach a general understanding of their content, identifying units of meaning and the initial codes, classifying the initial codes into broader categories according to their similarities and differences, and determining the hidden content of the data [30].

Accordingly, immediately after each interview, the first author (BT) transcribed the interview, read and reread the transcript, and, after obtaining a general understanding of the content, executed an inductive analysis of the information.

During the reading stage, important paragraphs were read carefully line by line. Words, sentences, or paragraphs which had significance regarding burn patients' dignity were designated as semantic units. A code was assigned to each key paragraph or phrase.

Subsequently, the second author (MR) reviewed the transcripts and verified the semantic units and open codes. Possible disagreements over the semantic units and codes were resolved in a meeting attended by all the four researchers (BT, MR, CT and MF).

All categories were then classified according to the similarities and differences of the codes. To ensure maximum strength of the codes, all the categories were revised and compared with the data. Next, in several meetings, the research team members (BT, MR, CT and MF) extracted the themes by careful and in-depth contemplation and comparison of the categories with each other.

In the processes of data collection and analysis, the researchers tried to apply bracketing. Bracketing is a method commonly used in qualitative research to mitigate the potentially undesirable effects of preconceptions that may cause bias in research [30]. Hence, the researchers attempted to ignore their 
own knowledge, beliefs, values, and experiences in order to accurately describe the participants' points of view on the concept of burn patients' dignity. The researchers did not form any judgment about the data and accepted them as they were. Data analysis was conducted using MAXQDA 2007 (VERBI ${ }^{\circledR}$ software).

\section{Study Rigor}

The accuracy and trustworthiness of the data were tested using Lincoln and Guba's criteria [31]. To ensure the credibility of the data, the researchers (1) applied prolonged engagement, member checking, peer debriefing. Before conducting the present study, the researcher (BT) was present in the wards as a nursing instructor and was regularly interacting with the participants in the course of the study. The collected data were reviewed with the participants (nurses and family caregivers) (2), triangulation (nurses and family caregivers of different genders and age groups), and maximum variation sampling (5) based on contrasting evidence. To confirm the dependability and conformability of the data, the researchers had a panel of experts examine the transcripts and the extracted codes and categories. The transferability of the results was ensured through complete and accurate descriptions of the topic of research, the participants' characteristics, and the method of data analysis, along with printed examples of the participants' statements.

\section{Results}

20 subjects ( 14 nurses and 6 family caregivers) participated in the present study. The age ranges of the nurses and family caregivers were 28-54 and 22-41 years respectively. The demographic characteristics of the participants are presented in Table 1.

Data analysis yielded three main themes: creating a friendly atmosphere, showing respect, and providing comprehensive support. The themes and their categories are shown in Table 2.

\section{Creating a friendly atmosphere}

One of the most important findings of the study, referred to by all the participants, was creating a friendly atmosphere. From the participants' perspective, providing high-quality care to patients while maintaining their dignity requires a cordial atmosphere between nurses, patients, and patients' families. By being kind to patients and dedicating time to them, nurses can build emotional intimacy with patients and impart a sense of comfort, trust, and respect to them. From the participants' perspective, intimacy with patients is the key to sympathizing with them and allows patients to discuss their problems with their nurses. This theme is comprised of 3 categories: empathy, friendly relationship, and dedicating time to patients. 


\section{Empathy}

From the viewpoint of the caregivers, empathy is of great importance in burns departments due to the special nature of burn injuries and the condition of the patients. Since burn patients' health and quality of life are usually very seriously affected by their injuries and they are hospitalized for longer periods, nurses have more time to be empathetic to the patients. This type of relationship helps nurses understand patients better and support them more effectively. Every burn patient needs physical and psychosocial care tailored to his/her specific needs. Regarding showing empathy for burn patients, one participant stated that:

"A burn patient needs a lot of empathy and emotional closeness; in fact, empathy is complementary to our treatment. No matter how good the care we provide is, it is not going to work without empathy..." (P1).

From the nurses' point of view, burn patients are less likely to complain of pain when they realize that nurses empathize with them and, thus, nurses can help patients more effectively.

"When I imagine myself in his [a burn patient's] position, it helps me a lot to understand his issues and concerns, and then I can help him more ..." (P6).

From the participants' point of view, good communication skills, including speaking clearly, using facial expressions, making eye contact, and listening attentively, play a major role in healing and reducing burn patients' suffering and increasing their satisfaction. Also, the participants believed that burn patients can express their concerns more freely when nurses establish an effective non-verbal communication with them.

"I hold my patients' hands, sit next to them, listen to and smile at them, all of which will raise their spirits and allow them to talk about their worries ..." (P8).

\section{Friendly relationship}

According to the interviews, burn patients need care which is combined with kindness and compassion. This kind of care makes the patients feel valued and believe that their dignity is being maintained.

"I start a friendly relationship with my patients and try to make them feel emotionally close to me. This makes them feel they are valued..." (P2).

The participants stated that, due to burn patients' prolonged length of stay, the patients feel more comfortable with the caregivers who call them by their first names, laugh with them, and treat them like a friend.

"I am very friendly to them and speak gently with them. I call them by their first names, and when we call them by their first names, they become happy and a friendly atmosphere is created" (P14). 


\section{Dedicating time to the patients}

According to the interviews with the study participants, burn patients feel more intimate and talk more freely when caregivers spend time with them. This also motivates patients to cooperate in their treatment. On the other hand, nurses' failure to spend quality time with their patients creates feelings of worthlessness and indignity in the patients.

"Sometimes, I talk to my patients for a long time about their problems and my own experiences .... When the patients realize how much time I am spending with them, they feel valued..." (P10).

\section{Showing respect}

From the caregivers' viewpoint, the traumatic experience of suffering a burn, painful hospital treatments, body dysfunctions, and expressions of pity from others cause burn patients to feel that they are not treated with dignity. Nurses can preserve the dignity of burn patients during care by showing respect for their identity and autonomy and by involving the patients in their treatment plans. The theme of showing respect consists of the categories of respect for human equality, respect for autonomy, respect for beliefs and values, and avoidance of pity.

\section{Respect for human equality}

One of the major challenges of preserving the dignity of burn patients while providing care to them is cultural differences. The population of Iran is comprised of various racial and ethnic groups. The majority of the population is Fars, but caregivers are likely to meet burn patients from Baloch, Azeri, Turk, Kurd, Lur, or Arab minorities. The existence of varieties in patients' cultural, ethnic, and social backgrounds can affect the sense of value of the patients and their families.

The participants of the present study stated that all patients are equal. Accordingly, caregivers must preserve the dignity of all patients, regardless of their gender, religion, race, ethnicity, economic status, and social class. One of the nurses stated that:

"Most of the patients here are from the poor classes of the society, but we give them all the services they need, regardless of their ethnicity or social class ..." (P9).

A family caregiver said:

"When a nurse provides care fairly to all patients, no matter what the cause of burn, be it self-immolation or an accident, this reflects the preservation of human dignity..." (P15).

\section{Autonomy}


The participants identified patients' participation in the decision-making and treatment process as an expression of respect for the patients' dignity.

"It is the worst kind of disrespect to give a burn patient no explanation. I involve my patients in their treatment plans, which makes them want to cooperate with me..." (P5).

The participants mentioned that, to preserve the dignity of burn patients, they provide them with information on the treatment processes and introduce them to similar patient cases which can help them make informed decisions.

"Some patients resist skin grafting; then I show them patients who have had skin grafting and introduce them to some of the patients who have been treated successfully. In fact, by giving them this information, I help the patients make better decisions about their treatment..." (P7).

\section{Respect for beliefs and values}

According to the participants, burn patients tend to become interested in mystical, spiritual, and religious practices. By providing prayer services, arranging meetings with a priest for them, and providing access to prayer books, caregivers can show respect for patients' dignity. One participant said:

Many patients here believe in praying. To respect the dignity of these patients, we've provided them with a bookshelf full of prayer books and this has really helped with their recovery ..." (P5).

\section{Avoidance of pity}

The caregivers in the present study mentioned that, though burn injuries can cause serious changes in burn patients' physical functions and appearances, the patients should be treated as ordinary people and the treatment team should avoid pitying behaviors. Showing undue pity for a burn patient is regarded as undermining the patient's dignity.

"Burns patients do not need to be pitied, and yet some nurses pity them. For instance, they say stuff like "Oh, poor thing!"... This can make the patient feel disrespected ..." (P5).

On the same note, the experiences of the family caregivers underscored the importance of avoiding pitying behaviors in the preservation of the patient's dignity.

"Burn victims are scared of being pitied by the people around them; this is disrespectful to these patients ..." (P16).

\section{Comprehensive support}

The findings of the study indicate that comprehensive support is an important contributory factor in maintaining the burn patients' dignity. Comprehensive support consists of the following categories: pain relief, emotional support, psychological support, and social support. 


\section{Pain relief}

The majority of the interviewed nurses referred to pain as the most important physical cause of complaint amongst burn patients. The slightest movement or the implementation of various treatment procedures can increase patients' pain. The participants stated that, since burn patients may suffer from multiple injuries and functional disabilities, relieving the intensity of their pain using different methods and regular evaluations of the efficacy of the measures taken to manage their pain demonstrate respect for the dignity of the patients. According to a nurse:

"We have a lot of patients who have lost their organs or suffered spinal cord injuries due to electrocution, and most of them are constantly complaining of pain. Replacing a burn dressing is a really painful experience. We use partial anesthesia when we want to replace a patient's dressing. After the procedure, if the patient is still in pain, painkillers are administered. We may use non-pharmacological interventions, too ..." (P12).

\section{Psychological support}

From the perspective of the study participants, burn injuries not only affect the patients' bodies, but affect the psychological health of the patients and their families. Burn patients desperately need the support of the people around them: because the treatment procedures are relatively long, are implemented in stages, and can be very painful, the patients may suffer depression, isolation, anxiety, humiliation, and loss of dignity. One of the most important aspects of nursing burn patients is providing them with the emotional and psychological support required to preserve their dignity and facilitate their recovery.

"Once, we had a patient who was seriously ill and her husband would not allow her family to visit her. I did my best to convince her husband to change his mind .... After the patient saw her family members, she felt very valued and recovered quickly..." (P1).

Based on the experiences of the caregivers of burn patients, it is important to consider the origin of the patients' mental distress and make an effort to resolve it toward maintaining their dignity.

"We have patients here who are scared of death. Family visits can help them a lot to cope with this fear. For these patients, we assign a round-the-clock companion..." (P4).

Other measures which can help preserve burn patients' dignity are: evaluating their mental and emotional well-being, providing them with counseling services, and ensuring collaboration between the members of the burn care team and the patients' families.

"Drug Addiction, family problems, and fear of alienation from the family and society are leading psychological problems in burn patients. We try to reduce the patients' psychological distress by raising 
their awareness, arranging counseling sessions for the patients and their families, removing the gaps between the patients and their family members, and facilitating cooperation between the treatment team and the patients' families ..." (P11).

Another nurse stated:

"After a patient is discharged, we introduce him or her to a counseling center where they and their families can receive counseling services ..." (P13).

\section{Social support}

According to the information gathered from the interviews, another major problem of burn patients is the social issues which they may encounter after the incident. Divorce, family disintegration, the society's negative attitude towards these patients, and the patient's decision to quit school or work are among the social harms which undermine burn patients' dignity. According to the experiences of the caregivers, the distressful nature of burn injuries and the associated complications may damage the ego of the victims.

A participant stated:

"We've had many patients who resigned from their jobs because of the inappropriate behaviors of their co-workers ..." (P12).

From the viewpoint of the caregivers interviewed in the present study, the financial support provided by insurance companies, charity institutes, and social welfare organizations is quite limited and burn patients view their nurses and family caregivers as the main sources of support for them. Therefore, nurses and social workers seek to preserve the patients' social dignity by helping them learn job skills and find jobs and by referring them to charity centers to help them pay their treatment costs.

"We had a patient here whose spouse separated from her because of her facial burn marks and then she had no source of income and came to us. Here, the personnel try and help out these people any way they can: we introduce them to charities, find them jobs, buy their medication for them ...." (P10).

\section{Discussion}

The results of the present study show that nurses' and family caregivers' perception of burn patients' dignity consists of three main themes: creating a friendly atmosphere, showing respect, and providing comprehensive support. According to the results of the interviews, empathizing with burn patients in a friendly relationship and dedicating time to them create a friendly atmosphere. In such a context, nurses can preserve the dignity of the patients by showing respect for the patients' intrinsic values and autonomy and attempting to meet their emotional, psychological, and social needs.

In the present study, the theme of creating a friendly atmosphere is comprised of the categories of empathy, friendly relationships, and dedicating time to patients. Nurses attempt to form a deep human 
and spiritual connection with their patients so that they can imagine themselves in their situation to preserve their dignity. Martins et al. (2014) have performed a continuous assessment of nurses' emotions when they are faced with patients' pain and distress. Nurses often identify with the pain and distress of patients and their family members [32]. In a study by Badger and Royse (2012), nurses are found to attempt to empathize with patients and their family members by listening attentively and trying to understand burn patients' experiences and concerns [33]. The results of another study demonstrate that, if patients find caregivers unapproachable and unfriendly, they feel insecure and consider their behaviors as disrespectful [16]. Gallagher et al. (2008) report that inadequate time dedicated to talking to patients, lack of eye contact, and negligence make patients feel worthless and humiliated [34]. Most studies emphasize the importance of communicating with patients in an honest and friendly way to discover their feelings and solve their problems [35]. Use of communication skills, such as effective use of silence, posture, tone of voice, eye contact, listening and smiling, and speaking while care is being provided reflect a sense of confirmation and understanding on the part of nurses $[26,35]$.

According to Watson, a human is a being who deserves to be cared for, respected, nourished, understood, and helped [36]. Watson believes that an ethical interpersonal relationship in a clinical environment indicates respect for human dignity. Nurses' ability to correctly identify and understand patients' feelings and relate to them is evidenced in their effective presence, openness, purposeful mental efforts, words and behaviors, body language, emotions, reasoning, and intuition [6].

The findings also revealed that creating a friendly atmosphere combined with respect and compassion gives patients a chance to discuss their problems with nurses with comfort and a sense of security. Nurses are more likely to fathom out patients' feelings, concerns, and insights and preserve their dignity by spending time with them.

Based on the experiences of the study participants, respect plays a significant role in maintaining the dignity of burn patients. The theme of showing respect consists of the categories of respect for human equality, autonomy, respect for beliefs and values, and avoidance of pity. From the participants' point of view, in order for the dignity of burn patients to be preserved, the patients must be provided with fair and unbiased care combined with respect for their beliefs and their autonomy.

Similarly, several studies emphasize the necessity of respect for the intrinsic value of humanity and the fact that dignity should not be a function of such factors as age, wealth, education, and severity of a patient's illness [37-38]. In one study, avoidance of differentiation between patients is introduced as one of the main indexes of professional values in nursing [39]. According to Baillie (2009), all human beings have an equal right to dignity which is to be acquired and cannot be taken away [40]. Matiti and Trorey (2008) mention that patients expect nurses to maintain their dignity, regardless of their social classes or health conditions [41].

The participants of the present study also stated that, due to their prolonged treatment processes and various complications associated with their injuries, burn patients and their family members feel more valued if they can participate in the decision-making processes and treatment plans. 
Pepastaro et al. (2016) report that patients' dignity is maintained when patients are involved in the making of medical decisions which are related to them [42]. Likewise, Baillie and Matiti (2013) conclude that observance of patient autonomy is a fundamental principle of patient-centered care, resulting in the preservation of patients' dignity [43].

In most Asian cultures, collectivism lies at the core of the common social belief system-in a collectivist culture, an individual's world view is influenced by the society and individuals perceive themselves as entities attached to the society. However, in North American and Western cultures in general, individualism prevails. In an individualistic culture, independence is preserved through every individual's discovery and expression of his/her unique characteristics, while a collectivist culture emphasizes the presence of every one, complying, and having a coordinated relationship with others [44-45]. In collectivist societies, including Iran, families should be involved in the decision-making processes of their patients. Though patients are at the center of the consultation meetings about their treatment plans, their families should also, according to the patients' preferences, be allowed to participate in the making of decisions.

Based on the experience of the study participants, attention to and respect for the beliefs and wishes of burn patients in Iran stand for respect for the dignity of these patients. In other studies, too, respect for patients' values is considered as essential to preserving their dignity. In some studies, respect for patients' values and beliefs has been reported as a component of professional patient-centered care $[22,46]$. According to one study, attention to patients' beliefs and thoughts is closely connected with patient dignity [47]. The concept of human dignity is dependent on cultural context. In all areas of healthcare, caregivers should respect the value systems of the patients, be aware of their cultural orientations, and learn about their cultural perspectives on health and sickness [48].

Cultural and religious values influence individuals' beliefs about human dignity and, thus, have an impact on patients' understanding of dignity in healthcare [47]. The participants' stressing the importance of showing respect for patients' values can be attributed to the religious beliefs of the patients and their families, all of whom were Shia Muslims: Islam dictates that all humans deserve to be respected. In other words, the tradition of respecting others in Iran may be rooted in the collectivism and cultural values of Iranians.

According to the experiences of the caregivers, the dignity of burn patients may be undermined by expressions of undue pity from the members of treatment teams or other people nearby. The results of the interviews showed that, sometimes, the members of treatment teams or burn patients' companions unintentionally cause more pain and suffering to the patients, rather than soothing them, by pitying behaviors.

Similarly, Mohammadi et al. (2020) and Bagherian et al. (2019) report that the dignity of patients is preserved when they are not pitied $[49,22]$. Compassionate care, which emphasizes human and ethical values, has recently become central to codes of ethics. Compassionate care is a deep feeling stemming from a connection to human suffering and is a moral response to it [27]. It seems that some caregivers 
are not familiar with compassionate care and only show pity to patients without any practical solutions, which behavior upsets patients and their companions.

Comprehensive support, another theme of burn patients' dignity, is comprised of the categories of pain relief, emotional support, psychological support, and social support. The caregivers interviewed in the present study emphasized the importance of comprehensive support in preserving the dignity of burn patients in Iran. From the participants' point of view, the destructive and traumatic effects of burn injuries affect all aspects of the patients' existence. Most of the caregivers reported pain as the most serious physical problem burn patients experience. Similarly, the results of another study show that all burn patients suffer from daily pains [50] and the experience remains in their memories forever [51]. In this situation, alleviating the patients' pain and meeting their other needs are the main responsibilities of nurses and a sign of respect for patients' rights. Burn patients often need nurses to support them with proper pain management [52]. Pain management is an ethical responsibility of caregivers and an essential element in nurses' professional codes of ethics [53]. In a study, burn survivors are found to view pain management and anxiety management, especially at the first replacement of their dressings, as crucial [54]. However, the experiences of most burn patients indicate poor pain management techniques on the part of caregivers [55].

According to the results of the interviews with the caregivers, burn injuries affect not only the patients' bodies, but also their mental health, family members, family relationships, and social activities (e.g. participation in social activities, employment, and education). In some cases, divorce and other catastrophic life changes have followed burn patients' hospitalization due to their injuries. According to the experiences of the caregivers, the devastating nature of burn injuries and the ensuing complications damage the ego of burn victims. The results of a study conducted in Iran show that burn survivors experience threats to all dimensions of their "self" in the form of disturbances in their feelings, cognition, sense of identity, and behaviors. These emotional-cognitive disturbances cause burn survivors to experience "self-disruption." Most of the participants in that study reported that they were able to overcome their hopelessness through their belief in God and receiving support from the people around them [56]. Several studies conducted in other countries also suggest that family support can facilitate burn patients' adaptation, improve their quality of life, promote their mental rehabilitation, and help them cope with their depression [4,57]. Sideli et al. (2010) report that people who get less social support are more likely to endure higher levels of stress and pain [24]. Some studies have confirmed the positive effect of peer support on the recovery and rehabilitation of burn patients [58-59]. One study (2015) indicates that nurses' ethical commitment to burn patients is demonstrated in the guidance and support which they provide to the patients, which, in turn, helps with patient participation and maintaining patient autonomy [53].

According to one of the few studies on the role of family support in patient dignity [48], though caregivers believe in holistic care, most of them focus exclusively on pain relief [27]. In the Iranian culture, the family is the main source of emotional support for patients; thus, the presence of family members and relatives at the side of patients is part of patients' regular care and their families' social and religious values. In

Page 15/23 
this culture, a holistic healthcare system dictates that professional caregivers should tend to the needs of patients, as well as their families, in the hospital environment [60].

One of the limitations of the present study is that the perception of dignity was determined via semistructured interviews with the caregivers of burn patients. For more accurate results, the researchers suggest interviews with other healthcare team members and patients. Another limitation is the data collection method: here, data were collected through semi-structured, individual interviews, while other methods of data collection may provide richer information on the dignity of burn patients. Therefore, it is suggested that future studies evaluate the experiences of other members of healthcare teams, as well as those of burn patients themselves, regarding dignity and employ other methods of data collection, including observation and focus group interviews.

\section{Conclusion}

Respect for patients' rights and dignity has been emphasized as one of the ethical responsibilities of professional caregivers. The dignity of burn patients is potentially at risk due to numerous physical, psychological, and social factors. The results of the present study confirm that preserving the dignity of burn patients produces positive outcomes. In order for the dignity of burn patients to be preserved, the patients should be respected and provided with comprehensive care in a friendly atmosphere. The results of the study can help healthcare managers and policy-makers to create a supportive environment in which the burn patients' dignity is effectively preserved. An empathetic relationship, dedicating time to listening to patients' words, showing respect for their beliefs and values, providing care free of discrimination, and giving comprehensive support were found to be influential in maintaining the dignity of burn patients.

\section{Declarations}

\section{Ethics approval and consent}

The present study has been approved by the Research Ethics Committee of Shiraz University of Medical Sciences, Shiraz, Iran (Code: IR.SUMS.REC. 1396.S197). Before being interviewed, the participants were informed about the objectives of the study, the confidentiality of their information, the reasons why the interviews had to be recorded, and the researcher's role. Written and oral informed consent was obtained from all the participants. The participants were also informed that they were free to withdraw at any stage of the study. The time and location of the interviews were selected at the participants' convenience.

\section{Consent to publish}

Not applicable.

\section{Availability of data and materials}


The interview data will not be shared since the participants have been guaranteed full anonymity.

\section{Competing interests}

The author(s) declared no potential conflicts of interest for the research, authorship, and/or publication of this article.

\section{Funding}

The author(s) received no financial support for the research, authorship, and/or publication of this article.

\section{Authors' Contributions}

BT made a substantial contribution to the acquisition of data, analysis, and interpretation of the data. MR and CT made a substantial contribution to the examination of the concept and design, data analysis, interpretation of the data, and revising the article critically before announcing it to be fit for publication.

MF has made a substantial contribution to the study design and revising the article critically.

MG has reviewed the article critically. All the authors have read and approved the final version of the manuscript.

\section{Acknowledgements}

The present article is part of a research project approved by Shiraz University of Medical Sciences, Southwest of Iran. The authors would like to express their gratitude to all the nurses of the burns wards of the teaching hospitals affiliated with the above-mentioned university and all the family caregivers who participated for their cooperation.

\section{Authors' Information}

${ }^{1}$ Community-based Psychiatric Care Research Center, Department of Nursing, School of Nursing and Midwifery, Shiraz University of Medical Sciences, Shiraz, Iran. ${ }^{2}$ Community-based Psychiatric Care Research Center, School of Nursing and Midwifery, Department of Nursing, Shiraz University of Medical Sciences, Shiraz, Iran. ${ }^{3}$ Community-based Psychiatric Care Research Center, Department of Nursing, School of Nursing and Midwifery, Shiraz University of Medical Sciences, Shiraz, Iran. ${ }^{4}$ Department of Epidemiology, Shiraz University of Medical Sciences, Shiraz. ${ }^{5}$ School of Health Nursing and Midwifery, University of the West of Scotland, Paisley, Scotland. mark.gillespie@uws.ac.uk 


\section{References}

1. Shirkhoda M, Kaviani Far K, Narouie B, et al. Epidemiology and evaluation of 1073 burn patients in the southeast of Iran. SEMJ 2011; 12: 11-21, http://emedicalj.com/en/articles/78475.html

2. Zamanzadeh V, Valizadeh L, Lotfı $\mathrm{M}$, et al. Preserving self-concept in the burn survivors: a qualitative study. Indian J. Palliat. Care 2015; 21: 182. doi: 10.4103/0973-1075.156492.

3. Outwater AH, Ismail H, Mgalilwa L, et al. Burns in Tanzania: morbidity and mortality, causes and risk factors: a review. Int J Burns Trauma 2013; 3: 18. PMID: 23386982, PMCID: PMC3560491

4. Moi AL, Vindenes HA and Gjengedal E. The experience of life after burn injury: a new bodily awareness. J Adv Nurs 2008; 64: 278-86. doi: 10.1111/j.1365-2648.2008.04807. x.

5. Smeltzer SC, Bare BG, Hinkle JL, et al. Brunner\& Suddarth's Textbook of Medical-Surgical Nursing. 12th ed. Philadelphia: Lippincott, Williams and Wilkins, 2010: 1719.

6. Watson Jean. Human caring science: a theory of nursing, 2nd ed. 2011; Jones \& Bartlett Learning co.

7. Manookian A, Cheraghi MA and Nasrabadi AN. Factors influencing patients' dignity: a qualitative study. Nurs Ethics 2014; 21: 323-34. doi: 10.1177/0969733013498526.

8. Kadivar M, Mardani-Hamooleh $\mathrm{M}$ and Kouhnavard M. Concept analysis of human dignity in patient care: Rodgers' evolutionary approach. J Med Ethics Hist Med 2018; 11. PMID: 30258554, PMCID: PMC6150922

9. Parandeh A, Khaghanizade M, Mohammadi E, et al. Nurses' human dignity in education and practice: An integrated literature review. IJNMR 2016; 21: 1. doi: 10.4103/1735-9066.174750

10. Mohammadi F, Rakhshan M, Molazem Z, et al. Caregivers' perception of dignity in teenagers with autism spectrum disorder. Nurs Ethics 2019; 26: 2035-46. doi: 10.1177/0969733018796679.

11. Berglund $B$, Anne-Cathrine $M$ and Randers I. Dignity not fully upheld when seeking health care: Experiences expressed by individuals suffering from Ehlers-Danlos syndrome. Disabil Rehabil 2010; 32: 1-7. doi: 10.3109/09638280903178407

12. Hall S, Goddard C, Speck PW, et al. "It makes you feel that somebody is out there caring": a qualitative study of intervention and control participants' perceptions of the benefits of taking part in an evaluation of dignity therapy for people with advanced cancer. J Pain Symptom Manage 2013; 45: 712-25. doi: 10.1016/j.jpainsymman.2012.03.009.

13. Bagheri $\mathrm{H}$, Yaghmaei $\mathrm{F}$, Ashktorab $\mathrm{T}$, et al. Patient dignity and its related factors in heart failure patients. Nurs Ethics 2012; 19: 316-27. https://doi.org/10.1177/0969733011425970

14. Borhani F, Abbaszadeh A and Rabori RM. Facilitators and threats to the patient dignity in hospitalized patients with heart diseases: a qualitative study. IJCBNM 2016; 4: 36. PMID: 26793729, PMCID: PMC4709810

15. Fernández-Sola C, Cortés MMD, Hernández-Padilla JM, Torres CJA, Terrón JMM and Granero-Molina J. Defining dignity in end-of-life care in the emergency department. Nurs Ethics 2017; 24: 20-32. doi: 


\section{$10.1177 / 0969733015604685$}

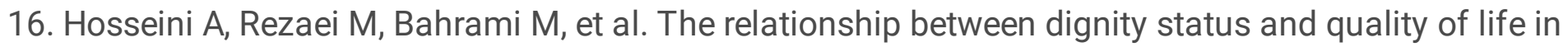
Iranian terminally ill patients with cancer. IJNMR 2017; 22: 178. doi: 10.4103/1735-9066.208157

17. Šaňáková Š and Čáp J. Dignity from the nurses' and older patients' perspective: a qualitative literature review. Nurs Ethics 2019; 26: 1292-309. doi: 10.1177/0969733017747960

18. Webster $C$ and Bryan K. Older people's views of dignity and how it can be promoted in a hospital environment. J Clin Nurs 2009; 18: 1784-92. doi: 10.1111/j.1365-2702. 2008.02674.x

19. Jamalimoghadam N, Yektatalab S, Momennasab M, et al. Hospitalized adolescents' perception of dignity: a qualitative study. Nurs Ethics 2019; 26: 728-37. doi: 10.1177/0969733017720828

20. Kostopoulou S, Parpa E, Tsilika E, et al. Advanced cancer patients' perceptions of dignity: the impact of psychologically distressing symptoms and preparatory grief. J Palliat Care 2018; 33: 88-94. doi: $10.1177 / 0825859718759882$

21. Avestan Z, Pakpour V, Rahmani A, et al. The correlation between respecting the dignity of cancer patients and the quality of nurse-patient communication. Indian J Palliat Care 2019; 25: 190. doi: 10.4103/IJPC.IJPC_46_18.

22. Bagherian $S$, Sharif $F$, Zarshenas $L$, et al. Cancer patients' perspectives on dignity in care. Nurs Ethics 2019. doi: $10.1177 / 0969733019845126$

23. Gerrek M, Behmer Hansen R and Khandelwal A. 440 Ethics in Burn Care: A Review. J Burn Care Res 2018; 39, Suppl (1): S192-S193. https://doi.org/10.1093/jbcr/iry006.362

24. Sideli L, Prestifilippo A, Di Benedetto B, et al. Quality of life, body image, and psychiatric complications in patients with a burn trauma: preliminary study of the Italian version of the Burn Specific Health Scale-Brief. Ann Burns Fire Disasters 2010; 23 (4): 171-176. PMID: 21991220, PMCID: PMC3188270

25. Baillie L, Gallagher A and Wainwright P. Defending dignity-challenges and opportunities for nursing. London: The Royal College of Nursing, 20 Cavendish Square, W1G ORN. Page: 20. https://www.dignityincare.org.uk/_assets/RCN_Digntiy_at_the_heart_of_everything_we_do.pdf

26. Ebrahimi H, Torabizadeh $\mathrm{C}$, Mohammadi E, et al. Patients' perception of dignity in Iranian healthcare settings: a qualitative content analysis. J Med Ethics 2012; 38: 723-8. oi: 10.1136/medethics-2011100396

27. Tehranineshat B, Rakhshan M, Torabizadeh C, et al. Nurses', patients', and family caregivers' perceptions of compassionate nursing care. Nurs Ethics 2019; 26: 1707-20. doi: $10.1177 / 0969733018777884$.

28. Elo S and Kyngäs H. The qualitative content analysis process. J Adv Nurs 2008; 62: 107-15. doi: 10.1111/j.1365-2648.2007.04569. x.

29. Hsieh HF, Shannon SE. Three approaches to qualitative content analysis. Qualitative health research. 2005;15(9):1277-88. doi: 10.1177/1049732305276687 
30. Graneheim UH and Lundman B. Qualitative content analysis in nursing research: concepts, procedures and measures to achieve trustworthiness. Nurse Educ Today 2004; 24: 105-12. doi: 10.1016/j.nedt.2003.10.001

31. Lincoln YS and Guba EG. Naturalist inquiry. Newbury Park, CA: Sage, 1985, p. 289.

32. Martins JT, Bobroff MCC, Ribeiro RP, et al. Feelings experienced by the nursing team at a burns treatment center. Escola Anna Nery 2014; 18: 522-6. http://dx.doi.org/10.5935/1414-8145.20140074

33. Badger K and Royse D. Describing compassionate care: The burn survivor's perspective. J Burn Care Res 2012; 33: 772-80. doi:10.1097/BCR.0b013e318254d30b

34. Gallagher A, Li S, Wainwright P, et al. Dignity in the care of older people-a review of the theoretical and empirical literature. BMC Nurs 2008; 7: 11. doi: 10.1186/1472-6955-7-11

35. Tehranineshat B, Rakhshan M, Torabizadeh C, et al. Compassionate Care in Healthcare Systems: A Systematic Review. J Natl Med Assoc 2019. doi: 10.1016/j.jnma.2019.04.002

36. Khademi M, Mohammadi E, Vanaki Z. Appraisal of the applicability of humanism based nursing theories. ijme. 2012; 5 (3):33-46. http://ijme.tums.ac.ir/article-1-116-en.html

37. Lin YP, Tsai YF and Chen. Dignity in care in the hospital setting from patients' perspectives in Taiwan: a descriptive qualitative study. J Clin Nurs 2011; 20: 794-801. doi: 10.1111/j.1365-2702. 2010.03499.x

38. Shahriari M, Mohammadi E, Abbaszadeh A, et al. Perceived ethical values by Iranian nurses. Nurs Ethics 2012; 19: 30-44. doi: 10.1177/0969733011408169

39. Bijani M, Tehranineshat B and Torabizadeh C. Nurses', nursing students', and nursing instructors' perceptions of professional values: A comparative study. Nurs Ethics 2019; 26: 870-83. doi: 10.1177/0969733017727153

40. Baillie L. Patient dignity in an acute hospital setting: a case study. Int J Nurs Stud 2009; 46: 23-37. doi: 10.1016/j.jnurstu.2008.08.003

41. Matiti MR and Trorey GM. Patients' expectations of the maintenance of their dignity. J Clin Nurs 2008; 17: 2709-17. doi: 10.1111/j.1365-2702. 2008.02365.x

42. Papastavrou E, Efstathiou $G$ and Andreou C. Nursing students' perceptions of patient dignity. Nurs Ethics 2016; 23: 92-103. doi: 10.1177/0969733014557136

43. Baillie L and Matiti M. Dignity, equality and diversity: an exploration of how discriminatory behaviour of healthcare workers affects patient dignity. Divers Equal Health Care 2013; 10. http://www.scopus.com/inward/record.url?

44. Ong-Flaherty C. Critical cultural awareness and diversity in nursing: a minority perspective. Nurse Leader. 2015 Oct 1;13(5):58-62. http://dx.doi.org/10.1016/j.mnl.2015.03.012.

45. Dai Q. Cross-Cultural Comparison of Self-Esteem among Mainland Chinese, Hong Kong Chinese, British-Born Chinese and White Scottish Children 2016; Social Science Asia, 2016 2(1): 1-12, doi: 10.14456/ssa.2016.3 
46. Mohammadi F, Tabatabaei Hs, Mozafari F, et al. Caregivers' perception of women's dignity in the delivery room: A qualitative study. Nurs Ethics 2019: 0969733019834975. doi: $10.1177 / 0969733019834975$

47. Cheraghi MA, Manookian A and Nasrabadi AN. Human dignity in religion-embedded cross-cultural nursing. Nurs Ethics 2014; 21: 916-28. https://doi.org/10.1177/0969733014521095

48. Hosseini FA, Momennasab M, Yektatalab S, et al. Patients' perception of dignity in Iranian general hospital settings. Nurs Ethics 2019; 26: 1777-90. doi: 10.1177/0969733018772078

49. Mohammadi F, Farjam M, Gholampour Y, Tehranineshat B, Oshvandi K, Bijani M. Health Professionals' Perception of Psychological Safety in Patients with Coronavirus (COVID-19). Risk Management and Healthcare Policy. 2020 Jul 14; 13:785-94. DOI: 10.2147/RMHP.S261410

50. Bayuo J, Agbenorku P and Amankwa R. Study on acute burn injury survivors and the associated issues J Acute Dis 2016; 5: 206-9. DOI: 10.1016/j.joad.2016.03.006

51. Shepherd $L$ and Begum R. Helping burn patients to look at their injuries: How confident are burn care staff and how often do they help? Burns 2014; 40: 1602-8. doi: 10.1016/j.burns.2014.02.017

52. Birdsall $\mathrm{C}$ and Weinberg K. Adult patients looking at their burn injuries for the first time. J Burn Care Rehabil 2001; 22: 360-4. doi: 10.1097/00004630-200109000-00015

53. Nogario ACD, Barlem ELD, Tomaschewski-Barlem JG, et al. Nursing Actions in practicing inpatient advocacy in a Burn Unit. Rev Esc Enferm USP 2015; 49: 0580-8. doi: 10.1590/S0080623420150000400007

54. Yuxiang L, Lingjun Z, Lu T, et al. Burn patients' experience of pain management: a qualitative study. Burns 2012; 38: 180-6. doi: 10.1016/j.burns.2011.09.006

55. Blakeney PE, Rosenberg L, Rosenberg M, et al. Psychosocial care of persons with severe burns. Burns 2008; 34: 433-40. doi: 10.1016/j.burns.2007.08.008

56. Zamanzadeh V, Valizadeh L, Lotfi M, et al. Self-disruption: Experiences of burn survivors. J Qual Res Health Sci 2014; 3: 269-80. file:///C:/Users/BlueSky/Downloads/Documents/jqr.ir-v3n3p269-en.pdf

57. Rossi LA, Costa MCS, Dantas RS, et al. Cultural meaning of quality of life: perspectives of Brazilian burn patients Disabil Rehabil 2009; 31: 712-9. doi: 10.1080/09638280802306257.

58. Badger K and Royse D. Adult burn survivors' views of peer support: a qualitative study. Soc Work Health Care 2010; 49: 299-313. doi: 10.1080/00981380903493095.

59. Öster $C$, Kildal M and Ekselius L. Return to work after burn injury: burn-injured individuals' perception of barriers and facilitators. J Burn Care Res 2010; 31: 540-50. doi: 10.1097/BCR.0b013e3181e4d692

60. Tabandeh S, Dehghan Nayeri N, Abbaszadeh A. Iranian Families' Experience of Receiving Support During Their Patients' Surgical Process: Qualitative Study. J NURS RES 2014; 22 (4): 268-27. 49doi: 10.1097/jnr.0000000000000055

\section{Tables}


Table 1 The themes and categories of this study

\begin{tabular}{|c|c|}
\hline Themes & Category \\
\hline \multirow[t]{3}{*}{ Creating a friendly atmosphere } & Empathy \\
\hline & Friendly relationship \\
\hline & Dedicating time to the patients \\
\hline \multirow[t]{4}{*}{ Showing respect } & Respect for human equality \\
\hline & Autonomy \\
\hline & Respect for beliefs and values \\
\hline & Avoidance of pity \\
\hline \multirow[t]{3}{*}{ Comprehensive support } & Pain relief \\
\hline & Psychological support \\
\hline & Social support \\
\hline
\end{tabular}

Table 2 The demographic characteristics of the participants 


\begin{tabular}{|c|c|c|c|c|c|}
\hline No & Job & Position & Marital Status & Work Experience & Education Level \\
\hline P1 & Nurse & Staff nurse & Married & 15 & Bachelor \\
\hline P2 & Nurse & Staff nurse & Single & 3 & Bachelor \\
\hline P3 & Nurse & Matron & Single & 28 & Bachelor \\
\hline $\mathbf{P 4}$ & Nurse & Head nurse & Married & 22 & Bachelor \\
\hline P5 & Nurse & Staff nurse & Married & 8 & Bachelor \\
\hline P6 & Nurse & Supervisor & Single & 24 & Master \\
\hline P7 & Nurse & Staff nurse & Single & 26 & Bachelor \\
\hline P8 & Nurse & Supervisor & Married & 18 & Master \\
\hline P9 & Nurse & Staff nurse & Single & 2 & Bachelor \\
\hline P10 & Nurse & Staff nurse & Single & 10 & Bachelor \\
\hline P11 & Nurse & Staff nurse & Married & 6 & Bachelor \\
\hline P12 & Nurse & Staff nurse & Married & 24 & Bachelor \\
\hline P13 & Nurse & Staff nurse & Married & 4 & Bachelor \\
\hline P14 & Nurse & Staff nurse & Single & 2 & Bachelor \\
\hline P15 & Family Caregivers & Housewife & Single & - & Diploma \\
\hline P16 & Family Caregivers & Housewife & Single & - & Diploma \\
\hline P17 & Family Caregivers & Employee & Married & - & Bachelor \\
\hline P18 & Family Caregivers & Self-employed & Married & - & Illiterate \\
\hline P19 & Family Caregivers & Self-employed & Single & - & Diploma \\
\hline P20 & Family Caregivers & Housewife & Married & - & Illiterate \\
\hline
\end{tabular}

\section{Supplementary Files}

This is a list of supplementary files associated with this preprint. Click to download.

- StandardsforReportingQualitativeResearch.docx 